\title{
Ambiente virtual de aprendizagem: estruturação de roteiro para curso online
}

Virtual learning environment: script structure of an online course

Ambiente virtual de aprendizaje: estructuración de guión para curso online

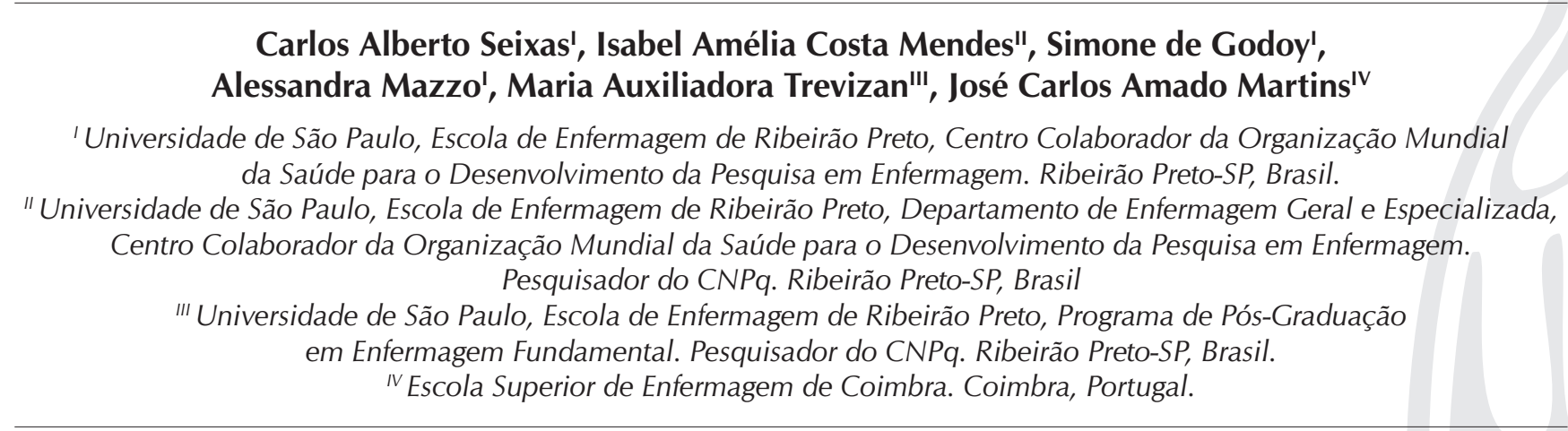

Submissão: 29-07-2011 Aprovação: 16-08-2012

RESUMO

O presente trabalho visa descrever os passos para desenvolvimento de um curso e sua estrutura em ambiente virtual de aprendizagem Moodle. Para tanto, a pesquisa consistiu na aplicação de conteúdos de enfermagem para oferecimento de curso online em workshop internacional para grupo de estudantes de graduação e licenciatura em enfermagem do Brasil e de Portugal. Durante a pesquisa foram registradas etapas distintas, desde o planejamento do curso passando pela construção e transformação dos conteúdos, até a disponibilização aos estudantes. As atividades interativas e conteúdos foram elaborados pelos professores com participação de equipe técnica. No trabalho são apresentados procedimentos específicos e papéis a serem desempenhados por professores, especialistas, estudantes e técnicos. Os resultados do desenvolvimento e oferecimento do curso online apontaram alguns aspectos a serem aperfeiçoados no processo de trabalho, no formato dos conteúdos e na utilização das ferramentas.

Descritores: Educação a Distância; Aprendizagem; Interface Usuário-Computador; Enfermagem; Tecnologia.

\section{ABSTRACT}

This study describes the steps for developing a course and its structure in the Virtual Learning Environment Moodle. It consisted of an application of nursing content offered in an online course included in an international workshop directed to a group of nursing students from a bachelor and a teaching diploma program in Brazil and Portugal. Distinct stages were identified during the study: planning, construction and transformation of content as well as availability of such content to students. The interactive activities and context were developed by professors with the participation of the technical team. The specific procedures and roles performed by professors, specialists, students and technicians are presented. The results of the development and offering of the online course appointed some aspects to be improved in the work process such as the format of content and use of tools. Key words: Education; Distance; Learning; User-Computer Interface; Nursing; Technology.

\section{RESUMEN}

La finalidad de este trabajo es describir los pasos para desarrollo de un curso y su estructura en ambiente virtual de aprendizaje Moodle. Para eso, la investigación comprendió la aplicación de contenidos de enfermería para ofrecimiento de curso online en oficina internacional para grupo de alumnos de pregrado y licenciatura en enfermería de Brasil y Portugal. Durante la investigación fueron registradas etapas distintas, desde la planificación del curso, incluyendo la construcción y transformación de contenidos, hasta su disponibilidad para los alumnos. Las actividades interactivas y contenidos fueron elaborados por los profesores con participación de equipo técnico. En el trabajo son presentados procedimientos y papeles que deben desempeñar los profesores, especialistas, alumnos y técnicos. Los resultados del desarrollo y ofrecimiento del curso online indicaron algunos aspectos que se debe perfeccionar en el proceso de trabajo, formato de los contenidos y en la utilización de las herramientas. Palabras clave: Educación a Distancia; Aprendizaje; Interfaz Usuario-Computador; Enfermería; Tecnología.

Extraído da Tese de Doutorado "Estrutura e dinâmica de curso em ambiente virtual de aprendizagem", apresentada, em 2011, à Universidade de São Paulo, Escola de Enfermagem de Ribeirão Preto. Ribeirão Preto-SP, Brasil.

\section{AUTOR CORRESPONDENTEＣarlos Alberto SeixasＥ-mail: seixas@usp.br}




\section{INTRODUÇÃO}

Historicamente, o processo ensino-aprendizagem é abordado de acordo com as mudanças que ocorrem em cada época na sociedade, por isso sempre é um tema atual. É definido como um processo dinâmico e contínuo de construção do conhecimento, desenvolvido livremente pela reflexão, pela interação entre os atores e levando a transformação da realidade ${ }^{(1)}$.

A educação está se configurando como um dos principais setores econômicos, ao lado da agricultura, da indústria. As novas tecnologias tem permitido a criação de ambientes educacionais multissensoriais, com estruturas diferenciadas (jogos, simulações, colaboração a distância, entre outras) e com capacidade de capitalizar o acesso ao conhecimento. A educação continuada compreendida como a busca crescente pelo aperfeiçoamento das pessoas desde a mais tenra idade até os seus últimos dias, está presente hoje em todos os setores e, portanto, alvo de estudos de educadores e pesquisadores de diversas áreas em todo o mundo ${ }^{(2)}$.

Neste contexto, a educação a distância (EaD), torna-se um dos elementos centrais dessa educação continuada, posto que permite alcançar um maior número de participantes em curto espaço de tempo e em diferentes localidades geograficamente distantes e com realidades culturais distintas, tornando-se uma ferramenta estratégica para sobrevivência dos profissionais ${ }^{(3)}$.

No entanto, no cenário atual da educação superior no Brasil nos cursos de EaD são constatados altos índices de evasão. Os materiais produzidos nem sempre apresentam adequação ao modelo online. As instituições reconhecidamente de excelência em seus programas presencias, apesar de disporem de infra-estrutura física e tecnológica adequada, não conseguem o mesmo sucesso, adesão e trabalho efetivo dos professores em suas ações na educação a distância ${ }^{(4)}$.

Os modelos de educação a distância vigentes incluem em sua formulação estrutura administrativa voltadas para ferramentas on-line, modelo pedagógico adaptado aos sistemas virtuais de comunicação e ferramentas tecnológicas próprias ${ }^{(5)}$.

Considerando que os atuais AVAS são softwares desenvolvidos para atendimento de públicos situados em diversas regiões do mundo, evidencia-se a necessidade de pesquisas voltadas para diferentes culturas e centradas na visão dos diferentes usuários em seus diversos papéis seja como conteudistas, professores, tutores ou estudantes ${ }^{(6)}$.

Uma das principais ferramentas utilizadas na educação a distância é o Ambiente Virtual de Aprendizagem (AVA) ou Virtual Learning Environment (VLE).

Na literatura é possível encontrar termos sinônimos como plataforma de educação a distância (PEaD), Learning Management System (LMS) e Course Management System (CMS).

A principal função do AVA é a de servir de repositório de conteúdos e meio de interação/comunicação entre os atores envolvidos no processo de ensino-aprendizagem ${ }^{(7)}$. Estes ambientes possuem diferentes formas de apresentação de suas ferramentas, com funções específicas e maneiras de distintas de interação com os usuários. Este conjunto de ferramentas pode ser subdividido em ferramentas síncronas e assíncronas.

As ferramentas assíncronas permitem acesso sem que haja necessidade de que todos os sujeitos estejam conectados simultaneamente. As ferramentas síncronas necessitam de conexão simultânea de todos os envolvidos na execução das atividades ${ }^{(8)}$.

Uma das formas de classificação de AVAs é feita através do modelo de distribuição de seu software. Nesta classificação pode-se dividi-los em duas categorias: os sistemas proprietários e os baseados em software livre. Os AVAS proprietários possuem distribuição baseada na comercialização de seu uso ou na venda como produto a ser implantado na empresa/instituição contratante. Normalmente seu código-fonte é fechado, ou seja, não permite reprogramação de suas ferramentas ou adaptações no funcionamento de seus recursos.

Os ambientes virtuais de aprendizagem baseados nos preceitos do software livre podem ser utilizados ou instalados gratuitamente e podem ser modificados/adaptados pelos programadores. Um dos exemplos de AVA baseado em código livre e aberto é o Modular Object-Oriented Dynamic Learning Environment (Moodle). A primeira versão do Moodle foi lançada no ano de 2001, pelo educador e cientista de computação, Martin Dougiamas, como parte de sua tese de doutorado na Curtin University of Technology, Austrália. Atualmente é aperfeiçoado e distribuído dentro da filosofia de software livre com colaboração de uma comunidade internacional de desenvolvimento, composta por técnicos, cientistas e programadores de todas as partes do mundo ${ }^{(9)}$.

O Moodle possui ferramentas presentes em outros AVAS, mas que podem ser ativadas e desativadas de acordo o planejamento estabelecido, podendo-se definir módulos aprendizagem com atividades e tarefas em formato colaborativo(7).

Os AVAs possuem características próprias mas, na maioria dos sistemas, são encontradas as ferramentas de interatividade similares com formato de apresentação distintos. Um dos sistemas usados na Escola de Enfermagem de Ribeirão Preto da Universidade de São Paulo (EERP-USP) é o AVA Teleduc desenvolvido pela Universidade Estadual de Campinas (UNICAMP). O AVA Teleduc, foi implantado na EERP-USP no ano de 2003, como uma das etapas do projeto de mestrado(10-11). Desde então tem sido utilizado como apoio ao ensino presencial nas disciplinas, tem sido satisfatório, visto que o perfil dos estudantes tem nível de conhecimento básico em informática, não foram identificadas dificuldades na operação de suas ferramentas e a equipe técnica de apoio é reduzida.

No entanto, para o uso de algumas ferramentas principalmente no oferecimento de exercícios e no processo inerente de obtenção dos resultados e posterior divulgação, a plataforma apresentou algumas limitações, o que levou a busca de novas alternativas de Ambientes Virtuais de Aprendizagem.

Iniciamos, portanto, o desenvolvimento de pesquisas em outras plataformas disponíveis para uso na Universidade de São Paulo como: Teleduc (CIRP), Cool (CCE), Sakay (Aprendizado Eletrônico) e Moodle (USP).

Com base nos testes de uso destes sistemas e nas experiências vivenciadas, os estudos passaram a explorar o uso do sistema Moodle (USP), mantido pela Universidade no campus de São Paulo.

O desenvolvimento dos trabalhos no Moodle (USP) não tem único propósito da substituição imediata do Teleduc, mas 
também em avançar nas pesquisas e oferecer alternativas de outras plataformas e de como melhor utilizá-las.

No levantamento da literatura pertinente e no âmbito da experiência vivenciada em grupo de pesquisas por mais de uma década, atuando em ações com a participação de professores, estudantes e pesquisadores interessados no uso destes ambientes e suas ferramentas, tem sido frequente o surgimento de dúvidas técnicas elementares sobre: como iniciar uso do ambiente, o processo de inscrição dos estudantes, forma de disponibilizar materiais, como devem ser os materiais, comportamento, número mínimo de acessos diários, como se comunicar com estudantes, quais ferramentas utilizar, como utilizar tais ferramentas, como definir prazos de entrega das atividades, como formular critérios de avaliação e como usar os critérios de avaliação.

Os estudantes, por sua vez, revelam algumas inquietações frequentes como: quando devem se inscrever na disciplina ou curso; se a matrícula da disciplina presencial já garante acesso ao AVA; quais credenciais utilizar para ingresso ao AVA; sobre as atividades e o prazo para entrega; sobre os locais e horários disponíveis para acesso ao AVA; como se comunicar com os professores e como entregar as atividades.

Estas questões revelam que há uma clara necessidade de orientação técnica para início do processo de utilização do AVA, mas, além disso, há também evidências que mostram uma demanda por serviços especializados que requerem a composição de equipe permanente que possa oferecer apoio técnico na produção e acompanhamento dos cursos oferecidos na modalidade a distância.

A comunidade estudantil estudada, por sua vez, formada em sua maioria por jovens entre 20 e 24 anos que já possuem hábitos de utilizar ferramentas virtuais desde a infância, demonstram necessidades e expectativas em encontrar ambientes similares em sua experiência no ensino superior. Vale salientar que estes estudantes já se comunicam com professores e colegas e fazem uso de conteúdos eletrônicos desde o ensino fundamental, através diversidade de dispositivos de hardware e software, inclusive usando computação móvel.

Revela-se ainda neste estudante a expectativa de que os instrumentos e ferramentas computacionais, apesar de ainda serem utilizados em pequena escala, ganhem recursos de interatividade, visibilidade, com melhores condições de acesso, como as ferramentas ágeis de comunicação utilizadas em seu universo de relacionamento pessoal como, por exemplo, os sistemas usados nas redes sociais. Os estudantes relatam sua insatisfação pelo pequeno oferecimento de conteúdos através de ferramentas modernas e também por encontrarem dificuldades de comunicação fora do espaço de aula entre os colegas e professores ${ }^{(12)}$.

Há, portanto, uma lacuna na produção e divulgação de conhecimento que leve em consideração este cenário onde coexistem profissionais e instituições de ensino interessados em fazer o melhor uso deste vasto ferramental disponível, mas que não conseguem alcançar resultados satisfatórios nem do ponto de vista dos estudantes e nem como mecanismo de melhoria de qualidade na educação.
No contexto deste estudo, optou-se por utilizar especificamente do ambiente Moodle, mas os momentos vivenciados no AVA Moodle também ocorrem no uso de outros AVAs, uma vez que há semelhança significativa no funcionamento dos processos de planejamento, inscrição, disponibilização, modelos avaliativos e acompanhamento da dinâmica dos cursos entre os diversos sistemas.

Nesta perspectiva propõe-se o aprofundamento em estudos que visem explorar o uso destes sistemas apoiados por equipe técnica especializada. Para tanto propõe-se mapear o processo de construção e oferecimento do curso através da vivência e registro de todas as etapas do processo.

\section{METODOLOGIA}

Trata-se de estudo metodológico descritivo de delineamento transversal. Em estudos transversais os fenômenos são obtidos durante um período de coleta de dados e apropriados para descrever a situação, o status do fenômeno ${ }^{(13)}$.

Na definição do escopo para o estudo objetivou-se atingir a maioria dos estudantes de enfermagem das instituições participantes: Escola Superior de Enfermagem de Coimbra (ESENFC) e Escola de Enfermagem de Ribeirão Preto da Universidade de São Paulo (EERP-USP). Participaram da pesquisa estudantes brasileiros dos $2^{\circ}, 3^{\circ}, 4^{\circ}$ e $5^{\circ}$ anos dos cursos de bacharelado e licenciatura em enfermagem; e estudantes portugueses, de licenciatura em enfermagem do $2^{\circ}, 3^{\circ}$ e $4^{\circ}$ anos; que aceitaram o convite e estavam regularmente matriculados no curso.

O presente estudo obteve as autorizações dos Comitês de Ética em Pesquisa das instituições envolvidas conforme previsto na Resolução CNS 196/96, sob os protocolos n $1225 / 2010$ e 01-09/2010 e CONEP parecer 195/2011. Os estudantes foram convidados a participar do estudo e formalizaram o aceite por meio da assinatura do Termo de Consentimento Livre e Esclarecido. Foi mantido o anonimato e a liberdade de interrupção da participação dos estudantes em qualquer etapa de realização do estudo.

O trabalho foi desenvolvido de acordo com objetivos estabelecidos e para o seu cumprimento as atividades foram executadas em fases distintas:

\section{$\checkmark \quad$ Etapa 1 - Planejamento}

$\checkmark$ Etapa 2 - Construção/Transformação dos Conteúdos

$\checkmark$ Etapa 3 - Desenvolvimento da Dinâmica do Workshop

$\checkmark$ Etapa 4 -Conclusão e análise dos acessos dos estudantes

A seguir se descreve como cada uma dessas etapas foi executada para o cumprimento dos objetivos.

\section{Etapa 1 - Planejamento}

Nesta etapa foi construído o plano de ação que contemplou os seguintes aspectos: definição do escopo do projeto (local e população de estudo, aspectos éticos, prazos, conteúdos trabalhados ), definição de estratégias de oferecimento do conteúdo, métodos de registro das etapas de construção e disponibilização, métodos de avaliação dos resultados e as etapas de construção roteiro com de diretrizes técnicas. 


\section{Etapa 2 - Construção e Transformação dos Conteúdos}

Nesta etapa, professores, técnicos e especialistas trabalharam na composição dos conteúdos, na adaptação semântica para o português de Portugal, no processo de transformação e inserção no AVA. Este trabalho foi registrado e descrito identificando os processos e atores envolvidos.

\section{Etapa 3 - Desenvolvimento da Dinâmica do curso}

O oferecimento do curso online é compreendido como a etapa dinâmica onde todos os participantes devem interagir com o AVA. Os papéis e funções de cada um dos atores foram mapeados e descritos em função das atividades executadas.

Etapa 4 - Conclusão e análise dos acessos dos estudantes

Os resultados das respostas dos estudantes foram agrupados e apresentados resumidamente com vistas a apontar pontos negativos e positivos na visão dos estudantes. Trechos das respostas dos estudantes foram selecionados, transcritos e categorizados em opiniões positivas e negativas. Os dados foram discutidos com o objetivo de identificar futuras melhorias no uso do AVA.

\section{RESULTADOS E DISCUSSÃO}

Os resultados são apresentados de forma descritiva. Para desenvolvimento da pesquisa buscou-se vivenciar e descrever todas as ações envolvidas na preparação de um curso no AVA Moodle. Como temática utilizou-se o Workshop sobre Retenção Urinária ocorrido no ano de 2011 em convênio estabelecido entre EERP-USP ESENFC.

Apesar das características do curso serem distintas de um curso regular, os processos estão presentes em ambos os formatos, englobando todas as fases de desenvolvimento, desde o planejamento das atividades de ensino até o encerramento com o processo de avaliação, similarmente o que ocorre em uma disciplina.

As atividades desenvolvidas pela equipe de trabalho incluíram as etapas de preparação, dinâmica de oferecimento e conclusão apresentados nos quadros 1, 2, 3 e 4 .

O trabalho de planejamento foi realizado em curto espaço de tempo (10 dias úteis) e com a participação ativa de todos os membros da equipe. Durante as reuniões foram feitas definições de datas e horários levando-se em consideração também o calendário acadêmico de ambas as instituições, bem como a diferença de fuso-horário entre Ribeirão Preto e Coimbra.

O processo de planejamento foi realizado a contento não apresentando dificuldades técnicas. Todos os passos e definições do planejamento foram executados no prazo previsto.

Os conteúdos foram desenvolvidos pela equipe docente, por meio de ferramentas de software que já estavam habituados a utilizar, pelo fato de eliminar necessidade de treinamento, minimizar possíveis incompatibilidades entre versões e possibilitar maior produtividade no desenvolvimento.

A etapa de desenvolvimento dos conteúdos foi iniciada concomitantemente ao processo de abertura das inscrições, tendo sido concluído no prazo total de 30 dias. O trabalho colaborativo da equipe docente foi realizado com uso de e-mail entre professores da EERP-USP e Escola Superior de Enfermagem de Coimbra e com a realização de videoconferências, utilizando o sistema Skype, nos encontros com menor número de participantes, e nos encontros com grupos maiores foram utilizadas as estações de videoconferência de ambas instituições. Os modelos e formatos das aulas expositivas e guias didáticas das atividades foram estabelecidos em conjunto com os técnicos e corpo docente. Nesta etapa também foram executadas as adaptações linguísticas necessárias.

O desenvolvimento se deu de forma rápida e natural confirmando a estratégia de manter as ferramentas de uso cotidiano dos professores e conforme previsto na metodologia.

Durante o desenvolvimento das atividades notou-se que a criação dos cursos é ainda uma das etapas pouco conhecida pelos professores e técnicos locais e que há necessidade de melhor estruturação dos processos envolvidos neste trabalho. Outro aspecto identificado é que há necessidade de melhor divulgação e orientação sobre as formas de solicitação de cursos no AVA.

As informações transmitidas dos professores à equipe técnica foram identificadas durante o processo de criação do curso e contribuíram para a composição do roteiro proposto na etapa de criação do curso, cuja criação foi feita de forma rápida e sem ocorrência de erros. O administrador técnico obteve as informações necessárias junto aos professores e estas foram imediatamente utilizadas no processo de criação.

\begin{tabular}{|l|l|l|l|}
\hline \multicolumn{1}{|c|}{ Processo } & \multicolumn{1}{|c|}{ Ações desenvolvidas } & \multicolumn{1}{c|}{ Informações requeridas } & \multicolumn{1}{c|}{ Atores e funções } \\
\hline Planejamento & $\begin{array}{l}\text { Título, Agenda, Ementa, Programas, } \\
\text { requisitos e público-alvo }\end{array}$ & $\begin{array}{l}\text { Datas, conteúdos programáticos, } \\
\text { conhecimentos prévios necessários }\end{array}$ & $\begin{array}{l}\text { Professores: construção } \\
\text { Técnicos: participação } \\
\text { Tutores: participação }\end{array}$ \\
\hline $\begin{array}{l}\text { Desenvolvimento } \\
\text { dos conteúdos }\end{array}$ & $\begin{array}{l}\text { Aulas expositivas, textos } \\
\text { obrigatórios, textos complementares, } \\
\text { exercícios, textos de orientação, } \\
\text { bibliografia. }\end{array}$ & $\begin{array}{l}\text { Conteúdos das aulas expositivas, } \\
\text { lista de textos, fontes de referência }\end{array}$ & $\begin{array}{l}\text { Professores: produção } \\
\text { Técnicos: orientação }\end{array}$ \\
\hline
\end{tabular}

Quadro 1 - Ações desenvolvidas na etapa de planejamento e preparação do curso online sobre assistência de enfermagem: retenção urinária em 2011. 


\begin{tabular}{|l|l|l|l|}
\hline \multicolumn{1}{|c|}{ Processo } & \multicolumn{1}{|c|}{ Ações desenvolvidas } & Informações requeridas & Atores e Funções \\
\hline $\begin{array}{l}\text { Criação do curso no } \\
\text { AVA Moodle-USP }\end{array}$ & $\begin{array}{l}\text { Inserção do curso no AVA com } \\
\text { informações dos professores }\end{array}$ & Título e duração do curso & Técnicos: inserção \\
\hline $\begin{array}{l}\text { Transformação dos } \\
\text { conteúdos }\end{array}$ & $\begin{array}{l}\text { Elaboração dos formatos finais } \\
\text { para inserção no AVA }\end{array}$ & Arquivos produzidos pelos professores & $\begin{array}{l}\text { Técnicos: produção } \\
\text { Professor: orientação e verificação }\end{array}$ \\
\hline $\begin{array}{l}\text { Inscrição dos } \\
\text { professores e } \\
\text { tutores no AVA }\end{array}$ & $\begin{array}{l}\text { Preparo para recebimento de } \\
\text { inscrições automatizadas ou } \\
\text { inserção manual }\end{array}$ & $\begin{array}{l}\text { Forma de inscrição, período de abertura, } \\
\text { fechamento, lista de nomes e e-mails }\end{array}$ & $\begin{array}{l}\text { Técnicos: programação } \\
\text { Professores: verificação }\end{array}$ \\
\hline $\begin{array}{l}\text { Customização das } \\
\text { ferramentas e layout } \\
\text { no Moodle }\end{array}$ & $\begin{array}{l}\text { Elaboração dos aspectos visuais e } \\
\text { ferramentas disponiveis no AVA }\end{array}$ & $\begin{array}{l}\text { Cores, formato de apresentação e } \\
\text { ferramentas selecionadas }\end{array}$ & $\begin{array}{l}\text { Técnicos: programação } \\
\text { Professores: aprovação }\end{array}$ \\
\hline $\begin{array}{l}\text { Inserção dos } \\
\text { conteúdos no AVA }\end{array}$ & $\begin{array}{l}\text { Inclusão dos conteúdos no curso } \\
\text { criado no AVA }\end{array}$ & Conteúdos transformados e aprovados & $\begin{array}{l}\text { Técnicos: inserção } \\
\text { Professores: verificação e adaptação }\end{array}$ \\
\hline $\begin{array}{l}\text { Verificação e testes } \\
\text { de acesso }\end{array}$ & $\begin{array}{l}\text { Os conteúdos já inseridos são } \\
\text { testados para ativação }\end{array}$ & Conteúdos inseridos e aprovados no AVA & $\begin{array}{l}\text { Técnicos: testes e correção } \\
\text { Professores:testes e notificação }\end{array}$ \\
\hline
\end{tabular}

Quadro 2 - Ações desenvolvidas na etapa de construção e transformação dos conteúdos do curso online sobre assistência de enfermagem: retenção urinária em 2011.

No desenvolvimento do trabalho de transformação das aulas expositivas pensou-se no formato que cumprisse a função de informar de forma completa ao aluno e indicasse claramente conteúdos e caminhos recomendados, pois não haveria presença física do professor. Este desafio foi buscado através de inclusão de elementos de texto de orientação e adaptação da linguagem feita pelo professor tentando traduzir as falas em sala de aula por elementos contidos na própria apresentação.

Neste aspecto, constata-se que muitos projetos utilizam com sucesso o recurso de apresentação com narração sincronizada com resultados altamente satisfatórios do ponto de vista de transmissão do conhecimento ${ }^{(14)}$.

O trabalho de inserção dos conteúdos foi realizado principalmente pelo administrador técnico local. Observou-se, no decorrer do trabalho, a necessidade de informações adicionais para seu pleno desenvolvimento. Estas informações foram obtidas com os professores, durante o trabalho de inserção. Em alguns momentos foi necessário acesso e verificação em tempo real ao AVA em conjunto com os professores para realização de adequações. Na inserção de conteúdos foi necessário o diálogo com os professores para definição de informações complementares e seqüência correta dos conteúdos disponibilizados.

Outro aspecto peculiar neste curso foi o de conter, simultaneamente, conteúdos em duas versões distintas do mesmo idioma, o que demandou discussões sobre melhor forma de dispor os textos.

Para inserção dos links de acesso das leituras o administrador local utilizou a ferramenta "acrescentar rótulo", com opção de abertura de nova janela de navegador para cada link de leitura disponibilizado.

Uma maneira mais adequada de dispor as leituras seria a substituição pela ferramenta "acrescentar - recurso - link a um arquivo ou site". Desta forma seria possível verificar os acessos dos estudantes em cada texto disponibilizado.

Para o desenvolvimento do curso online seguiu-se modelo com princípios de design instrucional que considera o formato de apresentação e estilo de aprendizagem, estimula a reflexão e incluem planos de orientação aos estudantes ${ }^{(15-16)}$.

Para o processo de inscrição foi prevista a elaboração de formulário eletrônico. Considerou-se que o modelo utilizado foi bem aceito, pois os estudantes não registraram dificuldades no acesso.

No encerramento foram gerados os relatórios finais consolidados de notas e de frequência, para fins de registro. Para fins de análise futura propõe-se que estes relatórios sejam produzidos em formato de planilha e protegidos.

O local de armazenamento destes relatórios deve coincidir com a estrutura criada para acomodação do curso, os arquivos devem ser gravados nas estações de desenvolvimento do administrador local e copiados em equipamento servidor de arquivos da EERP-USP.

Além disso, o administrador técnico deve executar procedimento de cópia de segurança do curso para servidor local, utilizando a ferramenta de backup do AVA Moodle localizada no módulo de administração. A partir da geração destes arquivos, uma segunda cópia de segurança deve ser gerada em mídia DVD e disponibilizada para equipe docente.

Estes arquivos podem ser utilizados no futuro, caso haja necessidade de se criar novamente o evento ou em outro sítio onde o AVA Moodle esteja instalado. 


\begin{tabular}{|l|l|l|l|}
\hline \multicolumn{1}{|c|}{ Processo } & \multicolumn{1}{c|}{ Ações desenvolvidas } & \multicolumn{1}{c|}{ Informações requeridas } & \multicolumn{1}{c|}{ Atores e Funções } \\
\hline $\begin{array}{l}\text { Inscrição dos } \\
\text { estudantes }\end{array}$ & $\begin{array}{l}\text { Preparo para recebimento de } \\
\text { inscrições automatizadas ou } \\
\text { inserção manual }\end{array}$ & $\begin{array}{l}\text { Forma de inscrição e período de abertura } \\
\text { e fechamento } \\
\text { Lista de nomes e e-mails de estudantes }\end{array}$ & $\begin{array}{l}\text { Técnicos: programação } \\
\text { Professores: verificação }\end{array}$ \\
\hline $\begin{array}{l}\text { Oferecimento } \\
\text { do curso }\end{array}$ & $\begin{array}{l}\text { Ativação dos conteúdos, } \\
\text { notificações e correção dos } \\
\text { exercícios verificação do acesso e } \\
\text { avaliação final e relatórios }\end{array}$ & $\begin{array}{l}\text { Ações dos estudantes e professores } \\
\text { emissão de relatórios }\end{array}$ & $\begin{array}{l}\text { Tutores: acompanhamento e } \\
\text { resposta às dúvidas } \\
\text { Professores: correção, respostas às } \\
\text { duvidas e acompanhamento }\end{array}$ \\
\hline
\end{tabular}

Quadro 3 - Ações desenvolvidas na etapa de desenvolvimento da dinâmica do curso online sobre assistência de enfermagem: retenção urinária em 2011.

\begin{tabular}{|l|l|l|l|}
\hline \multicolumn{1}{|c|}{ Processo } & \multicolumn{1}{|c|}{ Ações desenvolvidas } & \multicolumn{1}{c|}{ Informações requeridas } & Atores e Funções \\
\hline Avaliações & $\begin{array}{l}\text { Realizar avaliações sobre } \\
\text { conteúdo e interface }\end{array}$ & $\begin{array}{l}\text { Atividades entregues e relatórios de } \\
\text { freqüência }\end{array}$ & $\begin{array}{l}\text { Técnicos: geração de relatórios } \\
\text { Professores: avaliação }\end{array}$ \\
\hline $\begin{array}{l}\text { Atividades de } \\
\text { encerramento }\end{array}$ & $\begin{array}{l}\text { Realiza rotinas de encerramento: } \\
\text { backup e relatórios }\end{array}$ & $\begin{array}{l}\text { Curso encerrado com fechamento das } \\
\text { avaliações }\end{array}$ & Técnicos: cópias e relatórios \\
\hline
\end{tabular}

Quadro 4 - Ações desenvolvidas para na etapa de conclusão do curso online sobre assistência de enfermagem: retenção urinária em 2011.

Os resultados de nosso estudo somam-se aos de outros estudiosos $^{(17-21)}$ que exploraram aspectos técnicos e pedagógicos de outro AVA, como conteúdo, interação, tempo de resposta e qualidade de interface.

Análises de usabilidade do AVA permitiram a produção e proposta de workflow e modelo de curso online para AVA Moodle(22).

\section{CONSIDERAÇÕES FINAIS}

Os resultados da pesquisa revelam que o processo de construção e disponibilização dos conteúdos pode seguir por diversos caminhos, pois há ampla possibilidade de customização e formas de uso do AVA Moodle. Na etapa de planejamento buscou-se identificar um desses caminhos e optou-se pelo uso de um número reduzido de recursos e ferramentas, com vistas a simplificar os procedimentos técnicos e maximizar a visibilidade e aproveitamento dos conteúdos pelos estudantes. Os objetivos foram plenamente alcançados, pois a vivência de uso do AVA pelos atores que participaram da pesquisa resultou em material rico em detalhes sobre os processos envolvidos e tarefas de cada membro da equipe de trabalho.

Espera-se que esta proposta de uso e estruturação de cursos online contribua para o efetivo uso do AVAs em instituições de ensino, seja como apoio ao ensino presencial, semi-presencial ou para oferecimento de cursos a distância.

O Grupo de Estudos e Pesquisas em Comunicação no Processo de Enfermagem tem realizado investigações nesta área e considera importante a realização de maior número de pesquisas centradas no elemento humano e com vistas à gestão de conhecimento para obtenção de melhores resultados na produção e disseminação do conhecimento.

\section{REFERÊNCIAS}

1. Paschoal, AS, Mantovani MF, Méier MJ. Percepção da educação permanente, continuada e em serviço para enfermeiros de um hospital de ensino. Rev Esc Enferm USP 2007;41(3):478-84.

2. Litto, FM. Prefácio. In: Guia Brasileiro de educação a distância. São Paulo: Esfera; 2000. p. 5-6.

3. Oliveira MAN. Educação à Distância como estratégia para a educação permanente em saúde: possibilidades e desafios. Rev Bras Enferm 2007;60(5):585-9.

4. Associação Brasileira de Educação a Distância. Censo EaD.br. São Paulo: Pearson Brasil; 2010.

5. Litto FM, Formiga M. Educação a distância: o estado da arte, São Paulo: Pearson Brasil; 2008. p. 462.

6. Silva EC. Educação a distância: ambientes digitais para o 
processo ensino-aprendizagem em enfermagem psiquiátrica. São Paulo. Tese [Doutorado em Enfermagem]- Escola de Enfermagem de Ribeirão Preto da Universidade de São Paulo; 2007.

7. Cole J, Foster H. Using Moodle: Teaching with the Popular Open Source Course Management System. 2. ed. Estados Unidos: O’ReiLLY; 2008.

8. Boulos MN, Taylor AD, Breton A. A synchronous communication experiment within an online distance learning program: a case study. Telemed J E Health 2005;11(5):583-93.

9. Dougiamas M. [homepage da internet]. Moodle: opensource software for producing internet-based courses; c2001-01. [acesso em 2010 set 10]. Disponível em: $<$ http://moodle.com/ >

10. Seixas CA, Mendes IAC. E-learning e educação a distância: guia prático para implantação e uso de sistemas abertos. São Paulo: Atlas; 2006.

11. Seixas, CA. Implantação de plataforma aberta de educação a distância e sua aplicabilidade no contexto da enfermagem. São Paulo. Dissertação [Mestrado em Enfermagem]- Escola de Enfermagem de Ribeirão Preto da Universidade de São Paulo; 2005.

12. Paechter M, Maier B, Macher, D. Students' expectations of, and experiences in e-learning: their relation to learning achievements and course satisfaction. Comput Educ 2010;54(1):222-9.

13. Polit DF, Beck CT, Hungler BP. Fundamentos de Pesquisa em Enfermagem. Métodos, avaliação e utilização. 5. ed. Artmed; 2004, p.487
14. Rosenberg MJ. E-learning: strategies for delivering knowledge in the digital age. New York: McGraw-Hill; 2001.

15. Soine, S. Instructional Design in a Technological World: Fitting Learning Activities into the Larger Picture. In: ICALT 2001 ago 6-8. Madison, USA; 2001.

16. Lapolli F, Cruz CM, Motta CLR, Tolla CET. Modelo de Desenvolvimento de Objetos de Aprendizagem Baseado em Metodologias Ágeis e Scaffoldings. Rev Bras Infom Educ 2010;(18):2,17-32.

17. Telles Filho PCP, Cassiani SHB. Creation and evaluation cycle of a distance module for nursing undergraduates, named "Medication Administration". Rev Latino-Am Enferm 2008;16(1):78-85.

18. Rangel EML, et al. Avaliação, por graduandos de enfermagem, de ambiente virtual de aprendizagem para ensino de fisiologia endócrina. Acta Paul Enferm 2011;24(3):27-33.

19. Cogo ALP, Silveira DT, Pedro ENR, Tanaka RY, Catalan VM. Aprendizagem de sinais vitais utilizando objetos educacionais digitais: opinião de estudantes de enfermagem. Rev Gaúch Enferm 2010;31(3):435-41.

20. Cogo ALP, Silveira DT, Lírio AM, Severo, CL. A utilização de ambiente virtual de aprendizagem no ensino de suportes básico e avançado de vida. Rev Gaúch Enferm 2003;24(3):373-9.

21. Ribeiro MAS, Lopes MHBM. Desenvolvimento, aplicação e avaliação de um curso à distância sobre tratamento de feridas. Rev Latino-Am Enferm 2006;14(1):77-84.

22. Seixas CA. Estrutura e Dinâmica de Curso em Ambiente Virtual de Aprendizagem. São Paulo. Tese [Doutorado em Enfermagem]- Escola de Enfermagem de Ribeirão Preto da Universidade de São Paulo; 2011. 